Voix et Images

volxetimages

\title{
Va-et-vient et circularité de la rêverie chez Jean-Aubert Loranger
}

\section{Robert Giroux}

Volume 2, numéro 1, septembre 1976

Fernand Leduc

URI : https://id.erudit.org/iderudit/200022ar

DOI : https://doi.org/10.7202/200022ar

Aller au sommaire du numéro

Éditeur(s)

Les Presses de l'Université du Québec

ISSN

0318-9201 (imprimé)

1705-933X (numérique)

Découvrir la revue

Citer cet article

Giroux, R. (1976). Va-et-vient et circularité de la rêverie chez Jean-Aubert

Loranger. Voix et Images, 2(1), 71-91. https://doi.org/10.7202/200022ar d'utilisation que vous pouvez consulter en ligne.

https://apropos.erudit.org/fr/usagers/politique-dutilisation/ 


\title{
Va-et-vient et circularité de la rêverie chez Jean-Aubert Loranger
}

\begin{abstract}
J'ai fait mes études à une époque où «l'achat chez nous" subissait une éclipse. Nous étions tous tournés vers les U.S.A. La culture, pour moi, c'était Frank Sinatra, Fred Astair, Nelson Eddy, Charlie McCarthey, les comic strips, Hollywood, le Reader's Digest, etc. Les valeurs de culture, en tout cas, ne résidaient pas ici. Plus tard, j'aurais pu lire Nelligan; j'entendis en effet parler d'un poète québécois, auteur d'un sonnet, le «Bateau ivre"; mais je découvrais le vrai Rimbaud, puis Nerval, Breton, Eluard. II y a maintenant, et surtout en littérature, depuis 1963, une nouvelle crise de "l'achat chez nous", et, par voie de conséquences, surenchère des valeurs et des choses dites "d'ici ». De là peut être l'importance que l'on attribue actuellement à Nelligan. Quant à moi, je n'ai vraiment pas l'intention de commencer aujourd'hui à lire son œuvre...'
\end{abstract}

Jacques Godbout

Ce témoignage de l'écrivain Jacques Godbout à propos de l'œuvre hypostasiée de Nelligan pourrait être endossé par bon nombre de ses contemporains pour qui la valorisation de ce qui est production typiquement québécoise relevait d'une problématique arbitraire et restait tributaire d'une vie culturelle à la recherche d'elle-même. La génération d'écrivains et de critiques des années 50 ne jurait que par la France. Celle des années 60 était plutôt imbibée par la marée sourde des U.S.A. Et que dire de notre génération! Le problème reste ouvert et il vaut mieux laisser aux historiens ou aux soi-disants sociologues de la littérature le soin de démêler ce qui est à proprement parler d'ici ou d'ailleurs. Nous proposons dans les pages qui vont suivre une lecture synchronique des textes de Jean-Aubert Loranger. Ces textes n'étaient-ils pas tombés dans l'oubli depuis une cinquantaine d'années, à défaut d'une réédition, à défaut de lecteurs, à défaut peut-être aussi d'une vie et d'un destin aussi exemplaires que ceux de Nelligan. II aura sans doute manqué à Loranger d'être mort, comme le dit si bien Claude Péloquin, “en plein centre de la poésie ${ }^{2}$. Sortir les textes 
de Loranger de l'oubli, c'est à coup sûr tomber en plein arbitraire. Pourquoi ceux-ci plutôt que ceux d'A. de Bussières par exemple? D'autres que nous se sont déjà occupés à revaloriser l'œuvre poétique de l'auteur des Atmosphères, notamment Gilles Marcotte qui en a vanté la cmodernité ", et aussi Bernadette Guilmette dans une thèse non publiée: le Voyage intérieur de Jean-Aubert Loranger.

De ce poète de la nuit et de l'attente, du «grand désir effondré ", nous aimerions proposer une lecture naïve, un peu comme celle que proposait Gaston Bachelard à propos de tout vrai écrivain à l'écoute de ses rêveries profondes: "tout chez lui est rivière, dira Gilles Marcotte, chemin, fleuve, impérieux besoin de partir, de changer, de commencer [...]. Puisque le départ est impossible [...] il faudra lui faire place ici, dans l'immobilité du quotidien, découvrir la mer au bout du jardin, déployer l'immensité dans l'espace $\operatorname{clos}^{3}$ \%. C'est ce monde clos de l'intimité qui nous intéressera dans les lignes qui vont suivre. Comment un écrivain des années 1920-1940 arriva-t-il à circonscrire une intimité aussi limpide et aussi originale que la sienne, alors que ses contemporains de l'École littéraire de Montréal exception faite de A. Desrochers - s'évertuaient à parler de tout et de rien, sauf d'eux-mêmes et de la lourdeur du quotidien? Douleur lucide, solitude étouffante sans être affolante, tout chez Loranger rêve de la «partance définitive " à l'intérieur du cercle bien dessiné de la lampe et du silence:

Le kiosque est rond,

II est allumé

Par le milieu, et la nuit

D'autour colle aux vitres

Comme une noirceur de suie.

\author{
Et j'écris dans le kiosque, \\ Lanterne géante \\ Qui aurait beaucoup fumé. \\ - Parqué en mon rêve. \\ Je suis bordé de silence 4 .
}

Comme le dirait Jean-Pierre Richard, la critique nous paraît relever de l'ordre d'un parcours, non d'un regard ou d'une station. D'où la perspective psychologique de notre lecture. Notre intention n'est pas de faire voir si les textes de Loranger sont réussis ou non, riches ou pauvres. Que le lecteur reconnaisse et sache apprécier une rêverie vraie, cohérente et honnête, alors notre tâche n'aura pas été vaine. Signalons tout de suite que la thématique que nous avons réussi à circonscrire n'est pas uniquement une construction de sens déterminée par notre (despotique) discours critique. Notre analyse suit de très près la succession des textes de Loranger et l'échafaudage critique qui en est résulté obéit à la cohérence même du discours poétique, et s'en trouve par le fait même validé. 


\section{LA TRAVERSÉE ET LA MARCHE}

Le recueil des Atmosphères, publié en 1920, se compose de trois parties: six courts textes de prose poétique, Signets, encadrés par deux contes: le Passeur et le Vagabond.

Du Passeur ${ }^{5}$, conte très riche par ailleurs de fines allusions psychologiques et d'intéressants procédés narratifs, nous ne retiendrons que 1. la description topographique de l'espace ou lieu narratif, et 2. ce mouvement si caractéristique chez Loranger qui servira à définir: a) un métier, celui de "passeur»; b) une situation dramatique, celle de l'emprise soudaine de la vieillesse chez un homme simple et vigoureux; et c) un mode de rêverie, c'est-à-dire la prédominance de l'horizontalité, et plus tard de la circularité.

La description topographique du Passeur est fort simple et très précise: une rivière sépare deux rives; elle coule entre un village (rive basse) et une cabane de passeur qui se trouve de l'autre côté (rive escarpée). Cette rivière coule ainsi entre deux quais. Une seule rue traverse le village: elle se continue de l'autre côté de la rivière à travers un bois qui barre l'horizon; la barque ou le traversier du passeur sert de «trait d'union mobile des deux rives". D'un côté comme de l'autre de cette rivière, l'espace est fortement circonscrit: les horizons sont limités, comme bouchés. D'une part, le village avec ses maisons, son église, son moulin, ses cheminées d'usine ${ }^{6}$; et d'autre part, au fond d'une "grande plaine avec des moissons", un bois barre l'horizon; mais ce bois n'est pas impénétrable puisqu'il y a une route qui semble le traverser, sinon, qui prendrait la peine de traverser la rivière? Rien de précis n'est donné sur cet au-delà du bois: il semble tout de même justifier les mouvements et les fonctions des acteurs... Le narrateur précise même que la route vient du bois.

Cette localisation de la rêverie caractérise d'emblée un paysage conforme, nous allons le voir, à l'imaginaire de Loranger. Notons pour le moment qu'il ne s'agit pas d'une ville mais d'un petit village paisible et sans histoire; au lieu d'une forêt redoutable et impénétrable, le narrateur évoque l'existence d'un bois traversé d'une route; au lac, il préfère le mouvement tranquille d'une rivière. L'univers de Loranger est déjà un monde habité, doux, non hostile, sédentaire, silencieux et routinier... C'est aussi celui du conte intitulé le Vagabond?, là où un quêteux va de village en village pour quémander de quoi subsister. Et ce sera encore le monde du terroir qu'évoqueront les contes et nouvelles du Village, publiés en 1925 et sous-titrés: À la recherche du régionalisme. Même contexte villageois dans cette sorte de farce rustique qu'est l'Orage, dans le même recueil. Loranger obéit ainsi aux préceptes des membres de l'École littéraire de Montréal de l'époque. Même tendance dans les récits mieux réussis, réunis dans les Écrits du Canada français, et dont la date de publication remonte aux années 19371939.

Mais ce ne sont pas ces considérations historiques autour de l'école du terroir qui nous intéressent surtout. Nous voudrions plutôt insister sur une permanence de la rêverie de Loranger. Cette rapide description topo- 
graphique (géométrique) qui ouvre le Passeur nous amène à considérer un phénomène beaucoup plus large et beaucoup plus significatif. II s'agit d'un mouvement bien caractéristique de l'imagination de Loranger, celui du vaet-vient.

Ce mouvement d'aller-retour définit un métier et un type de comportement psychologique. Le va-et-vient caractérise bien sûr, le métier même du passeur, lui dont la fonction est inséparable de son instrument de travail, sa chaloupe, définie par «son va-et-vient de trait d'union mobile des deux rives". Bien plus, le passeur fait corps avec sa chaloupe et les passages abondent où les rames sont comme la prolongation naturelle des bras. C'est alors que ce mouvement de va-et-vient du passeur (et des villageois) arrive aussi, par homologie, à définir la situation dramatique dans laquelle il se trouvera brutalement plongé, à savoir la prise de conscience de l'usure irrémédiable de son corps (bras, reins) qui n'arrive plus à remplir sa fonction et le voue à l'inactivité.

La paralysie du passeur oblige en effet ce dernier à rêver à l'intérieur d'un espace encore plus réduit que celui que lui imposait son métier. Aux horizons bouchés du paysage et à l'itinéraire coutumier de la traversée correspondra le lieu restreint qu'imposera l' «ankylose» du vieillard: ce dernier est ainsi contraint d'aller de sa chaise au lit et du lit à sa chaise, les deux nouvelles bornes de ses déplacements physiques. Autour du vieillard quasi immobile et miné par l'ennui viennent alors tourner les journées et les saisons. Ce mouvement prend ainsi deux aspects selon qu'il s'agit de l'espace ou du temps. D'une rive à l'autre ou du lit à la chaise, l'aller-retour est toujours rêvé et vécu sur le plan de l'horizontalité. La verticalité ne sera évoquée qu'à l'occasion de la noyade du passeur, descente verticale et fatale aux fonds des eaux. Si l'on met à part cette caractéristique (verticalité) de la rêverie qui se confirmera aussi ailleurs, comme nous le verrons, il est justifiable de ranger Loranger parmi les rêveurs du type de SaintDenys Garneau plutôt que du type des Nelligan ou des François Hertel sans cesse tiraillés par le haut et par le bas. Chez Loranger, l'horizontalité domine et entraînera par voie de conséquence un tiraillement particulier du dedans et du dehors: la tension s'installe entre l'ici et l'ailleurs, entre l'immobilité et la marche par exemple, jamais entre ciel et terre. La rêverie de Loranger est donc bien peu théologique, et il est très important de le souligner et de se le rappeler si l'on ne veut pas la réduire à ce que la critique a trop souvent tendance à faire quand elle cherche à tout prix à reconnaître chez un écrivain quelconque les indices d'une rêverie d'époque ou d'une collectivité. Bref, ce mouvement de va-et-vient est horizontal quand il est question de l'espace et cyclique quand il sera question du temps, autour d'un point fixe, autour non de la mort mais d'une ankylose comme la vieillesse, la paralysie, l'hiver, etc. Sans entrer dans les détails des variations de la rêverie du mouvement chez Loranger, en faisant intervenir par exemple le mouvement caractéristique du rameur assis (la rotation des bras... puis le glissement sur les eaux ou le même geste comme repoussoir de la mort), les quelques remarques qui précèdent au sujet du mouvement de va-et- 
vient obsédant d'un personnage nous introduisent à ce qu'il faudra bien considérer comme un mode d'être du rêveur/narrateur lui-même dans sa relation d'objet et sa relation d'autrui ${ }^{8}$.

Si l'on examine ensuite le conte intitulé le Vagabond et que l'on s'intéresse au personnage que le narrateur observe et à la signification que cette figure prend par rapport à l'imaginaire de Loranger, on constate que l'on ne retrouve plus ce mouvement de va-et-vient comme dans le Passeur ou, plus tard, dans le Retour de l'enfant prodigue. Il y a plutôt un simple mouvement vers l'avant, une marche qui va d'un village hostile au village suivant afin de venger une humiliation. En fait, si l'on s'en tient au mouvement seulement, le conte peut se diviser en deux parties: a) mouvement vers l'avant, b) attente immobile. Marcher, cela est dit explicitement, c'est se fatiguer, épuiser en soi, «par la distance», l'énergie nécessaire à la réalisation d'un projet de vengeance ou de révolte. En réalité, la marche va au contraire exagérer et amplifier le besoin de voler (double sens) quelqu'un au village suivant. La marche a donc un équivalent psychologique identique à la situation d'immobilité ou d'attente qui caractérise la deuxième partie du conte. En effet, que le vagabond marche ou qu'il soit en situation d'attente, l'intensité de sa rêverie s'accroît considérablement, tout comme le rêveur, en hiver, sent s'amplifier en lui, progressivement, le désir d'une débâcle et d'un départ possible...

Le vagabond va alors se faufiler sournoisement dans la cour d'une maison un peu à l'écart des autres. Et là, il attend la nuit: au fur et à mesure que la nuit s'épaissit, son attente devient "fiévreuse" et il prend peur devant le "trop-plein de force" qu'il éprouve, devant cette "poussée fiévreuse qui donnait à ses mains une envie d'étranglement ».

Dans le deuxième poème des Signets, Les hommes qui passent, le narrateur se met aussi en situation d'attente et, témoin passif, il constate que "la rue existe"; elle est en effet progressivement envahie par le bruit des tambours et le rythme des passants. A mesure que le bruit se rapproche et s'amplifie, le narrateur éprouve que la rue "se concentre et se retrouve", qu'elle "s'accorde" au rythme des pas et des tambours, qu'elle "se pénètre" et acquiert ainsi un mode d'existence. Même phénomène ici dans le Vagabond, mais avec plus de violence et d'angoisse. Le narrateur raconte que le vagabond souffre de son attente prolongée pendant laquelle la nuit l'envahit et l'oblige à se concentrer et à se retrouver (tel qu'en lui-même) : «La nuit en s'épaississant lui devenait intérieure.» “ll se mit à craindre cette nuit qu'il allait devenir."

Les poèmes de Signets ne sont autres que la reprise thématique, en sourdine ou en mineur, de ce que révèle avec beaucoup d'intensité la marche puis l'attente immobile du vagabond ${ }^{9}$. II faudrait longuement commenter Je regarde dehors par la fenêtre et surtout Je marche la nuit dans la rue où se retrouve explicitement évoquée la figure du voyageur, de l'étranger, du vagabond:

Je marche la nuit dans la rue, comme en un corridor, le long des portes closes, aux façades des maisons. 
Mon cœur est dévasté comme un corridor, où il y a beaucoup de portes, beaucoup de portes, des portes closes ${ }^{10}$.

Remarquons aussi que dans Des gens sur un banc ${ }^{11}$, Loranger ne commente qu'un seul mouvement, celui du va-et-vient des yeux des voyageurs en attente du train dans une gare: leurs yeux vont du tableau horaire à leur valise, en silence; assis, immobiles, dans "une même vie d'attente, ils ne sont rien qu'unanimes ».

Avec l'Hiver soudain ${ }^{12}$, quatrième texte des Signets, nous retrouvons les mêmes thèmes de l'engourdissement, de la paralysie et de la rêverie sur place que dans le Passeur. Le passeur subissait en effet l'hiver comme un arrêt de la vie: la rivière gelée, il s'enfermait immobile dans sa maison; il hibernait. Dans le poème ici, l'hiver n'évoque pas que l'ennui et la congélation. Il est certes question du «port en deuil», silencieux, déserté par les bateaux sur une eau qui s'épaissit puis se fige. Toutefois, si l'hiver symbolise l'arrêt et le gel, il est surtout un stimulant positif de rêverie. Nous entrons ici en pleine intimité: décembre n'est pas un temps mais un lieu: "Voici décembre par où se fait la fin de l'illusion qu'il y avait en moi d'une possibilité de partir. " Dans la glace, immobile, le narrateur s'abandonne à la fin d'une illusion, se résigne à toute possibilité de départ qui le faisait vivre quand le port était en activité.

\section{L'ATTENTE}

Par rapport à Nelligan, la rêverie de Loranger paraît bien passive et quelque peu terne. C'est que Loranger prend l'attitude par excellence d'un narrateur, d'un témoin qui raconte et fait voir "ce grand sillage que l'hiver garde matérialisé ${ }^{12} \%$, donnant une sorte de permanence congelée à un désir de départ rendu impossible. Nous remarquions plus haut que la figure du passeur se définissait dans son mouvement de va-et-vient horizontal. La paralysie physique ou l'ennui hivernal (la paralysie, la vieillesse et l'ennui sont à l'hiver et à la glace, ce que la vitalité, la jeunesse et le travail sont à l'été, l'eau libre et mouvante) se caractérisait par un mouvement cyclique du temps et de la rêverie, comme chez la petite Yolande, la petite infirme des Miraculeuses matines ${ }^{13}$. La rêverie poétique arrivera ainsi à se définir pour Loranger comme une tristesse qui se recueille sur elle-même, qui se ferme, telle celle des "veilleurs de feux établis sur les cimes" de Terra Nova ${ }^{14}$. Le va-et-vient est ainsi à la dérive, ce que l'inaction est au cercle, et plus matériellement, comme nous le verrons, aux objets ronds et/ou creux. Donc, double localisation du narrateur chez Loranger, deux situations antithétiques bien propres à caractériser l'ambivalence de la rêverie: l'attitude de l'observateur passif d'une part, comme nous venons de le voir, et d'autre part, l'attitude active du narrateur qui se déplace lui-même au milieu d'un monde figé et étranger. Quand il se tient immobile, le narrateur observe les gens dans la rue et tout semble alors s'animer, l'observateur n'ayant plus qu'à commenter ce qui vit devant lui. 
Passeur ou vagabond, narrateur immobile ou en marche, la rêverie s'éprouve toujours en situation d'attente, rêverie autour d'un nœud de conscience, jusqu'à ce que le désir s'amplifie, jusqu'à ce que la débâcle devienne imminente et fasse peur tout à coup, provoque un mouvement de recul, un renoncement à un désir trop grand... mais avec l'espoir de le reprendre puisqu'il le fait exister (comme la rue, la vie), puisque ce désir le place dans un mode d'être qui est son unique raison d'écrire et de vivre. $\mathrm{Au}$ cœur de cette rêverie qui tourne en rond, le rêveur se concentre et se retrouve en un aller-retour continu. Et comme les grandes cheminées du port du cinquième poème des Signets, il se mire dans l'eau qui mire...

\section{LES DÉSIRS DU VOYAGE IMPOSSIBLE}

Le deuxième recueil de textes que Loranger publia en 1922 s'intitule tout simplement Poèmes. II se compose aussi de trois parties: a) Préliminaires et Marines, b) Moments (Haikas et Outas), et c) le Retour de l'enfant prodigue. La partie centrale est constituée de très courts poèmes qui rappellent une certaine technique orientale de composition et d'inspiration; les vers ne comptent pas plus de sept syllabes et l'allure d'ensemble de chacun des textes annonce déjà l'aspect squelettique des Songes en équilibre que publiera A. Hébert en 1944.

Abstraction faite des procédés de composition qui sont par ailleurs très révolutionnaires pour l'époque au Québec, Berthelot Brunet faisait remarquer en 1922 que c'est tout le recueil qui aurait dû porter le titre: le Retour de l'enfant prodigue.

La mélancolie du départ, l'inutilité du voyage, les tristesses de la route, tout cela revient comme un leitmotiv.

Loranger a la hantise d'un départ définitif, il se décide à l'aumône de la clarté des lampes familiales dans la maison paisible, et il revient les yeux aveuglés de n'avoir vu que le noir et le vide. Après tant d'Invitation au voyage, après tant de Brises marines, il écrit une Invitation au retour $[\ldots]^{15}$.

Étudions d'abord la première partie du recueil, les poèmes réunis sous le titre: Marines. Avec Ébauche d'un départ définitif, nous entrons d'emblée dans la rêverie qui suit la débâcle printanière. Et les images du Passeur viennent quasi spontanément sous la plume de Loranger:

Le fleuve pousse à la mer

L'épaisse couche de glace

D'un long hiver engourdi,

Tel, avivé, repousse à

Ses pieds, le convalescent

Des draps habités d'angoisse ${ }^{16}$.

Remarquez que le narrateur n'est pas face à la mer. II est face au fleuve (rivière), sur le quai d'un port. Le mouvement d'aller-retour s'accorde ici au mouvement de flux-reflux de la mer. Encore une fois, la marée n'est pas 
évoquée comme étant haute ou basse; fidèle à une rêverie qui privilégie l'horizontalité, Loranger parlera du flux et du reflux de la mer dans le fleuve. Au reflux libérateur de la glace que le fleuve pousse vers la mer répondra le flux familier de

L'ancienne peine inutile

D'un grand désir d'évasion ${ }^{17}$.

Ce grand désir inutile ne fait qu'amplifier les distances, élargir l'espace devant..., confirmer et faire revivre une fois de plus la "forme mobile" du large (ou du vide intérieur). Malgré son refus catégorique de n'être qu'un "désemparé", il reste que sa rêverie se fait, clouée sur place: de ce point fixe, il contemple le (son) vide, en "mesure la distance" et refait "l'inverse de la jetée", revient à l'espace étroit de sa chambre, lieu de la conscience de l'immobilité des choses, lieu solitaire et vide de l'écriture.

Rappelons-nous la fin du Passeur: «les bras d'un moulin battaient l'air» (circularité); la barque du vieux passeur épuisé voguait entre les deux rives de la rivière, au gré du vent et du courant; puis la chaloupe se "retourne» (se renverse) pour ne laisser voir à la surface de l'eau que les "anneaux" du plongeon (de la noyade), circulaires ${ }^{18}$. La dernière strophe du poème est constituée des mêmes éléments, sauf que les «anneaux" deviennent ici le point de départ et que les rives vont s'élargir à la hauteur du golfe jusqu'à la mer... quand les anneaux vont lâcher prise:

Les cables tiennent encore

Aux anneaux de fer des quais [...]

Je partirai moi aussi.

J'enregistrerai sur le fleuve

La décision d'un tel sillage,

Qu'il faudra bien, le golfe atteint,

Que la parallèle des rives

S'ouvre comme deux grands bras,

Pour me donner enfin la mer ${ }^{19}$.

Le deuxième poème, intitulé le Port ${ }^{20}$, ramène impitoyablement la rêverie au rivage, ou plutôt, la rêverie revient sur elle-même, à son point de départ, sur les quais. Au lieu d'évoquer un départ hypothétique dans un futur indéterminé, le narrateur s'attarde plutôt au passé. Il relate mélancoliquement un souvenir:

Pourtant, je me souviens encore

De ce petit port au couchant,

Où mon rêve a voulu se plaire.

Cependant, cette complaisance dans la construction de son rêve ( « Pourtant...) ou cette projection du rêve sur l'écran d'un petit port ancien nous paraissent drôlement ambivalentes, sinon décadentes. "n'y a qu'à retenir les éléments de description de ce lieu où, dit-il, il faisait bon rêver: a) des filets étaient tendus sur les quais et dans les mâtures; ils «vieillissaient le fond de la crique comme des toiles d'araignées"; b) des barques étaient alignées le long de la jetée, "Sans casser leur ligne de file/ Pareille à un membre transi/Trop engourdi pour se détendre»; c) au 
lieu de s'attarder à une description éventuelle de marins pittoresques, Loranger s'attarde plutôt à évoquer une attitude d'esprit, «le meilleur d'eux-mêmes" que berce lentement le doux roulement des misaines, ce qui n'empêche pas le narrateur de sentir le besoin de rappeler, insistant toujours sur le décor un peu malsain de sa rêverie, "les rapaces désirs du gain" que les marins laissent dormir au plus profond des cales jusqu'à la marée haute du lendemain; d) viennent enfin s'ajouter deux annotations sonores et lumineuses: “Le trop-plein des sons alourdis/D'une heure lente et déjà vieille" tombant goutte à goutte du campanile de la ville, et les feux des phares qui s'allument dans le jour faiblissant.

Retenons les filets qui sont comme des toiles d'araignées; l'engourdissement de la ligne des barques; le bercement doux mais trompeur des fonds de cale; le trop-plein des sons alourdis à une heure lente et vieille de la journée; et enfin, le jour faiblissant. II n'y a en fait que les feux des phares qui ne soient pas évoqués dysphoriquement. Le narrateur ne les décrit pas. II ne fait que les nommer, constater leur présence. II dit qu'ils s'allument et on a l'impression qu'ils ne sont que les seuls à jouer un rôle actif dans le décor. Quel rôle ou quelle fonction peuvent bien remplir les phares dans la rêverie de Loranger?

Le poème suivant est éclairant à ce propos. II s'intitule précisément: les Phares ${ }^{21}$. Disons tout de suite que les ailes tournantes des moulins du Passeur trouvent leur équivalent imaginaire dans les phares, «ces moulins dont tournent les ailes lumineuses dans la nuit". Le moulin connote la présence du vent (ou de l'eau); le phare connote celle de la nuit et ses ailes sont des rayons de lumière sur la mer. Tous deux se caractérisent par leur tournoiement, double symbole dont se sert Loranger pour évoquer: a) le va-et-vient de sa hantise de départ; b) les «tournoiements alternatifs" de sa rêverie au couchant, les soirs de départ: i) un beau rêve qu'il harmonise dans un chant crépusculaire, "Espoir des choses lointaines"; ii) un "beau rêve effondré", "Extase triste" d'un soir de départ, "Où s'emplit d'ombre un vide immense", "Des distances douloureuses".

Le problème, c'est que l'alternance de la rêverie (va-et-vient ou départ-retour) se vit au même moment, se vit au cœur même de l'écriture qui raconte, et c'est ce qui fait toute la tension de chacun des courts textes de Loranger. La traversée dont rêve Loranger se résoud à une pure traversée scripturale, à une pure traversée qui constitue le texte même de sa rêverie.

O le beau rêve effondré

Que broient des meules d'angoisse

Dans les phares...

De la plus haute falaise.

Je regarde, dans la nuit,

D'autres phares sabrer l'ombre. 
C'est ce que l'on appelle communément le douloureux poétique, c'est-à-dire une tension qui ne se résoud qu'en un désir d'écriture, en un désir solitaire. Dans Ode ${ }^{22}$, le phare permettra en effet de prendre conscience de la profondeur de la solitude: l'ombre fera surgir en lui "comme le feu d'un projecteur". Et cela est d'autant plus douloureux qu'il sentira parfois le besoin de retrouver le parcours monotone mais sûr du passeur, retrouver le va-et-vient mesurable et contrôlable d'une vie circonscrite entre deux rives: par exemple,

Je voudrais être passeur;

Aller droit ma vie,

Sans jamais plus de dérive, Soumis à la force

Égale de mes deux bras.

Je voudrais être passeur; Ne plus fuir la vie ${ }^{23}$.

Ce motif des phares est très important pour Loranger et sera repris dans une même structure thématique dans Moments:

phare - moulin - ailes lumineuses dans la nuit;

phare - broyeur d'un grand désir effondré;

phare - «attente prolongée» - sabreur d'ombre (de mer).

Autant la mer se confond avec le vide et l'ombre durant la nuit, autant le brouillard se confond paradoxalement avec le plein, l'opaque, l'ombre, en plein jour. C'est ce qu'exprime clairement le troisième texte des Marines: le Brouillard ${ }^{24}$. Avec ce poème, nous sommes le matin, «ce triste et froid matin" au cours duquel Loranger va essayer de définir le brouillard. Pour lui, le brouillard n'est pas un élément vaporeux, délicat et mouvant. Au contraire, il est solide, il "solidifie l'air", il nous "recouvre, sans issue, en d'oppressantes voûtes froides". La distance perd tout son sens: lorsque l'on quitte le port, on peut mesurer la distance du sillage que laisse le bateau dans sa course vers l'avant. Mais lorsque l'on entre dans le brouillard, la distance que l'on voyait croître "vient sombrer au bout des yeux". La vue est comme bouchée: plus de sillage, plus d'horizon (l'horizon est à portée de la main, et une barque disparaît à portée de voix), plus de ciel puisqu'il devient obscurci et se métamorphose en «voútes froides"; il ne reste plus que soi ou un navire sur une surface liquide qui nous supporte, en une sorte de «rond-point" vide et neutre, sorte de degré zéro:

Et le bastingage a marqué

Le rond-point qu'assiège en exergue

L'inutile espace insondable ${ }^{25}$.

En ce "rond-point", tout sombre au bout des yeux. Le fond de l'espace nous est interdit, on ne le voit plus, mais "par delà l'opaque brouillard", on peut entendre des choses. Et voilà que Loranger reprend sa position d'homme en attente, attentif à la vie qui grouille autour de lui, même dans l'ombre, même dans le cercle bouché d'un brouillard, à la vie "dissimulée» dans le brouillard: “du fond de l'espace», il écoute les 
"graves cris alternatifs" d'autres paquebots dissimulés dans le brouillard et qui montent dans le ciel obscurci; et par delà, «l'inutile appel éploré des sirènes d'un sémaphore". Et lui, il est entre les deux, en un "rondpoint". Cela est constant chez Loranger: dans le brouillard, les mesures se font selon la portée de la voix (intensité variable du contact). Ailleurs le narrateur raconte une expérience des ténèbres:

J'avais perdu mes limites

Fondu que j'étais

Avec l'épaisseur de l'ombre.

Comme c'est pareil,

Ouvrir ou fermer les yeux ${ }^{26}$.

Que ce soit l'obscurité ou le brouillard, c'est toujours la même rêverie de l'enveloppement, du clos, du «rond-point», de l'opacité... Dans le premier “moment», Ébauche d'un départ définitif, l'ombre sera aussi rêvée comme une solidification, tel le brouillard. La lumière, au contraire, ce sera l'élément qui absorbera l'ombre et "dissolidifiera" l'espace: «La lumière absorbe l'ombre,/Elle dissolidifie/Le volume de la chambre ${ }^{27}$. Alors que la lumière est un élément de dispersion, l'ombre apparaît plutôt comme un élément de concentration, même dans la légèreté blafarde du brouillard. Le Journal de Saint-Denys Garneau fera écho, plus dramatiquement, à cette plainte solitaire:

J'accepte donc tout ceci comme une épreuve [...]. Réduit où j'en suis, qu'est-ce que je peux devenir? Je ne puis plus regarder en paix ni les arbres, ni les animaux; mes regards malades crèvent et souffrent partout où ils se heurtent, partout confrontés avec mon vide [...]. En dedans de moi, je ne trouve que le désert et le néant ${ }^{28}$.

Quoi qu'il en soit, ce motif de l'ombre et de la lumière reste ambivalent, et nous verrons plus loin que cette ambiguïté est liée au fait que ce motif varie selon qu'il s'agit d'une lumière ou d'une ombre qui appartient à l'extérieur (la nuit) ou à l'intérieur (la chambre close).

Le long poème méditatif du recueil, texte à l'intérieur duquel va venir se fondre toute la thématique que nous avons vue se développer jusqu'ici: c'est Ode ${ }^{29}$. Poème du désir et du renoncement, sorte de résignation lyrique, Ode exprime cette «plainte nostalgique» des pourquoi sans réponse. Pourquoi se plaindre que la brume efface les voiles du large, qu'un nuage décolore la mer, que la nuit efface tout l'horizon?

Pour une côte où brille un phare,

Pourquoi la plainte nostalgique,

Puisqu'à l'horizon le silence

A plus de poids que l'espace?

Pourquoi s'attrister du fait que le reflux de la marée ait laissé des voiles dans le (un) port? Pourquoi «pleurer l'impossible essor» et le grand désir irréalisé du large quand les départs manqués restent pour toujours ancrés en nos souvenirs: 
Tes yeux garderont du départ

Une inconsolable vision,

Mais à la poupe s'agrandit

Le désespoir et la distance.

Alors pourquoi se nourrir de l'illusion du jour, quand on sait très bien que le crépuscule va bientôt faire "s'effondrer" cette illusion ou ce désir de départ:

Et c'est bien en vain, que tu greffes

Sur la marche irrémédiable

De la nuit vers le crépuscule,

Le renoncement de tes gestes.

À la tombée du jour, le vieux port va rallumer ses lumières et apprendre deux choses que l'on sait déjà: la valeur, à l'horizon, du "jour enfoui" et la valeur du "rêve qu'édifie l'ombre». Entre ces deux valeurs, celle du jour et celle de la nuit, celle d'une nostalgie de départ d'aube impossible et celle du rêve de départ qui s'édifiera dans l'ombre, pourquoi ne préférerait-on pas celle de la nuit? Restons donc dans l'ombre, choisissons la nuit et le silence, et
Si l'ombre fait surgir en toi,
Comme le feu d'un projecteur,
Une connaissance plus grande
Encore de la solitude,
Que peux-tu espérer de l'aube?

C'est cette prise de conscience nocturne qui devrait occuper le lendemain, et non pas cette reprise d'un désir de départ, désir que l'on sait à l'avance inutile et impossible. Jamais l'azur du matin ne garde de trace des rayons tournoyants du phare: «l'espace où le phare a tourné » ne saurait être le même que l'espace de «l'azur», et dans l'un comme dans l'autre, le désir est stérile.

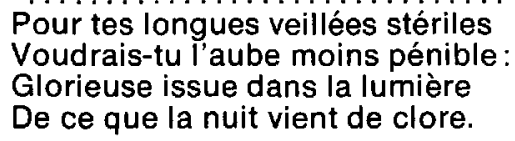

La rêverie ne pouvant se “clore» que la nuit, elle aura besoin de se «parquer" de silence, du grand silence qui "enclôt comme en une serre chaude où [la] peine doit mûrir». C'est précisément sur cette apologie du silence que vont insister les textes de la deuxième partie du recueil: Moments; tout se passe comme si après les rêveries marines, le narrateur se résignait à s'enfermer entre les quatre murs de sa chambre.

\section{RÊVERIE CIRCULAIRE DE LA CHAMBRE}

Le thème explicite de ces «moments" c'est le silence... Le thème implicite semble toutefois l'immobilité du rêveur dans une pièce close... La nuance est importante. Nous assisterons à la reprise de la situation de 
rêverie qui était celle du passeur paralysé, de même qu'à la reprise obsédante des motifs du clos, de la rondeur, du centre, etc.

Le premier «moment ${ }^{30}$ », c'est précisément celui de l'aube, moment sur lequel se fermait (ou s'ouvrait) le texte Ode. On s'attendrait à quelque éclaircissement sur cette “Glorieuse issue dans la lumière/De ce que la nuit vient de clore". Mais il n'en est rien. Le poète reprend obstinément la même rêverie nostalgique du silence qui «enclôt comme en une serre chaude où [sa] peine doit mûrir ". Il revient aussi sur le même souhait que dans Ode: il ne faudrait pas avoir attendu l'aube en vain, il faut qu'il y ait “le commencement aussi, de quelque chose... " Mais ce n'est qu'un vœu. II n'y aura pas de commencement, mais plutôt un recommencement de la rêverie, un encerclement. Le deuxième «moment» est très significatif à cet égard. II confirme deux choses: i) la passivité et l'immobilité du narrateur-témoin; ii) l'activité quasi agressive des éléments extérieurs qui l'obligent à recréer sans cesse cet état silencieux, solitaire et immobile dont il a besoin pour entretenir la fausse sérénité de sa rêverie: à l'occasion d'une averse, la «chambre sonore s'emplit d'une rumeur d'applaudissement». Pendant que le jour baisse, la lampe grandit, envahit la chambre et l'atteint (agressive) tel un projecteur que l'on braque sur un figurant «subitement confus, comme laissé seul et hué". Le silence est rompu, le rêve s'est dispersé: tout est à recommencer. Même rêverie au neuvième moment. Quand on a réussi à se fondre avec la nuit, «avec l'épaisseur de l'ombre", on perd ses limites: qu'importe alors d'ouvrir ou de fermer les yeux, "c'est pareil». "Mais le couloir s'allume» (agression de la lumière):

Ma chair oubliée

Se crispa, soudain touchée.

- Une aiguille claire,

Un rayon par la serrure ${ }^{31}$.

Le troisième «moment» est une autre rêverie immobile sur l'ombre, la lumière et le bruit. Mais contrairement au poème précédent, les éléments extérieurs ne seront pas agressifs; ils vont plutôt concourir à intensifier l'immobilité silencieuse. Le tic-tac de l'horloge remplace le bruit de la pluie du poème précédent; au lieu de rompre brutalement le silence,

L'horloge cogne sur le silence

Et le cloue, par petits coups,

A mon immobilité ${ }^{32}$.

De même que le silence est instauré, la nuit aussi sera amplifiée par son contraire: le bruit confirme le silence, tout comme la lumière de la lampe va confirmer la nuit. A l'extérieur de la chambre, c'est une «nuit d'ailleurs", celle du corridor obscur; la vraie nuit, c'est celle de l'intérieur, celle même que vient amplifier la lampe:

Des pointes d'ombre persistent

Attachées aux encoignures,

On dirait des découpures

D'une nuit encor plus vraie

Que la lampe a oubliées ${ }^{33}$. 
Le narrateur jouit donc d'une atmosphère qu'il a toujours souhaitée: nuit, silence, immobilité. Mais comme dans la plupart de ces courts poèmes, le sujet-narrateur s'introduit dans la dernière strophe. La description quasi objectale de la chambre nocturne prend alors une dimension affective: comme cela est fréquent chez Loranger, la réalisation d'un désir appelle un nouveau désir, souvent contraire au premier. L'immobilité silencieuse et nocturne de la chambre devient après coup oppressive (comme l'envahissement de la nuit chez le quêteux du conte intitulé le Vagabond), et le narrateur va se mettre à rêver non plus de la nuit «plus vraie" mais de la nuit extérieure, celle du corridor qui va le mener à la nuit du jardin:

Je sais que le jardin vit

Par plus de détails, ce soir,

Qu'en l'oppression de la chambre.

Sur l'étang ridé au vent,

La lune allongée s'ébroue

Comme un peuplier d'argent.

On peut interpréter cette dernière strophe selon deux visées: a) l'oppression de la chambre risque de devenir ennuyeuse et même intolérable. Vaut mieux alors se laisser distraire par le dehors, par le jardin qui «vit par plus de détails ": étang, vent, lune, peuplier, etc. On pourrait rappeler

La mer bruit au bout du jardin,

Comme l'orée d'une forêt... ${ }^{34}$.

Mais ces vers, nous les avons étudiés sous l'angle de celui qui se réfugie loin des quais pour mieux comprendre sa solitude; b) conformément à cette interprétation, le narrateur pourrait laisser entendre qu'il préfère "l'oppression de la chambre» à l'éparpillement (détails) de sa rêverie vers le dehors. Le narrateur réaffirmerait ainsi la nécessité de la solitude et de l'immobilité d'un lieu clos de rêverie - toute oppressante que soit cette situation. Les «moments" suivants semblent confirmer cette préférence et cette nécessité...

Le quatrième «moment ${ }^{35}$ ", ce sera en effet celui de l'heure «pleine", de la pleine mesure: minuit. Au cœur de la nuit, «les clochers rélèvent, au loin, les distances", "la nuit referme ses portes", et le scripteur écoute son cœur battre au cœur de sa chair (confirmation du va-et-vient de la distance et de la circularité de la durée). La majorité des autres textes seront dominés par cette thématique d'un centre de la conscience et d'une périphérie circonscrite, «parquée», «bornée», etc. Le cinquième "moment " est symptomatique à cet effet: il développe concrètement la thématique du centre et de la périphérie, non plus par le bruit de minuit ou des cloches, mais par la lumière et l'ombre (la suie). Le schéma de rêverie reste tout de même identique, sorte de va-et-vient entre intérieur et extérieur, dedans et dehors du kiosque rond allumé par le milieu. C'est ce poème que nous avons choisi pour clore l'introduction de notre propre texte ${ }^{36}$.

Comme on aura pu le remarquer, sur les dix-neuf textes de Moments, il y en a trois qui évoquent l'aube, quatre autres se situent durant le jour; 
la majorité des autres «moments" évoquent le soir et surtout la nuit, lieu d'écriture par excellence. Dans le Retour de l'enfant prodigue, un texte s'intitule Aube ${ }^{37}$ : des sabots cognent sur les pavés de la rue, la lampe se meurt, l'aube tarde trop et le narrateur en souffre puisqu'à l'aube son

\author{
[...] cœur inlassable \\ Dont je croyais tout savoir, \\ Revient doucement frapper \\ A la porte du rêve. \\ Toc, toc, toc.
}

Tout est à recommencer. Le désir renaît à l'aube. Partir! D'où le Retour de l'enfant prodigue, sorte de moralité prévisible. Avec un peu d'humour, B. Brunet écrivait: «Et vous verrez que, dans un autre volume, Loranger conclura comme les vieux sages repus et lourds d'expérience, qui conseillent aux jeunes fous de cultiver leur jardin ${ }^{38}$. " Le critique ne manquait pas de clairvoyance.

Toutefois, avant d'étudier cette troisième partie des Poèmes, il convient de résumer les observations qui nous ont semblé les plus pertinentes et propres à circonscrire le dynamisme intime de l'imagination de Loranger. La rêverie spatiale privilégie un mouvement, celui du va-et-vient. Elle fait intervenir deux figures masculines obsessionnelles: a) le passeur, sédentaire routinier; et b) le vagabond, nomade malheureux. Cette rêverie spatiale conditionne aussi une position de narrateur, celle d'un témoin, d'un observateur objectif en un point fixe, sur un quai, une falaise... Ailleurs, et de plus en plus, cette position sera celle de quelqu'un qui est en attente: ce qui déplace les rêveries spatiales sur le plan de la temporalité, l'espace se réduisant aux quatres murs d'une pièce, chambre ou kiosque, à l'intérieur de laquelle le narrateur écrit.

La rêverie temporelle insiste en effet sur la circularité de l'heure et des saisons, sur la circularité de la rêverie surtout: c'est que la rêverie tourne toujours en rond sur elle-même (départ-retour) et elle fait constamment référence à des objets ronds: tournoiement des phares, brouillard qui enclôt, chambre qui est comme une serre chaude où le rêve doit mûrir, etc. D'où cette tendance à préférer le silence à l'espace (l'oppression de la chambre plutôt que les «détails» extérieurs qui dispersent la rêverie) et la nuit à l'aube.

\title{
V. LE RETOUR : LÀ-BAS EST À ICI CE QUE DEMAIN EST A HIER
}

Dans le Retour de l'enfant prodigue, le titre marque un des inéluctables mythes auxquels, disait Mallarmé, ne peut échapper quiconque veut écrire. Ce recueil est constitué de six poèmes numérotés (dont le cinquième est la reprise du texte préliminaire des Poèmes) et de quatre poèmes titrés. Si Marines s'inscrit sous le signe de la résignation immobile et silencieuse, le Retour reprend le désir de partir et propose l'aventure du vagabond. "Ouvrez cette porte où je pleure ${ }^{39}$." Ce vers rappelle certes celui, célèbre, d'Apollinaire: «Ouvrez-moi cette porte où je frappe en pleu- 
rant." II rappelle surtout les poèmes où se manifeste la dialectique de la chambre et du couloir et aussi de la rue bordée de chaque côté par les portes closes des maisons de Je marche la nuit:

Je marche la nuit dans la rue, comme en un corridor, le long des portes closes, aux façades des maisons.

Mon cœur est dévasté comme un corridor, où il y a beaucoup de portes, beaucoup de portes, des portes closes ${ }^{40}$.

Mais ici, il est question d'une seule porte, "cette porte où je pleure", la porte devant laquelie le narrateur-sujet fait l'aumône de la clarté des lampes. C'est ce que raconte le premier groupe de vers qui seront repris à la fin du poème:

Ouvrez cette porte où je pleure.

La nuit s'infiltre dans mon âme

Où vient de s'éteindre l'espoir,

Et tant ressemble au vent ma plainte

Que les chiens n'ont pas aboyé.

Ouvrez-moi la porte, et me faites

Une aumône de la clarté

Où gît le bonheur sous vos lampes.

L'espoir, c'est la clarté des lampes, la chaleur humaine (bonheur), l'intimité. Les recherches illusoires, ce sont les ténèbres de la nuit, la plainte nocturne du vent, la solitude et le vide. D'où l'aumône, la quête de ce qu'il a toujours obstinément recherché. Mais ici intervient, implicitement, une autre personne, autrui comme confident ou aide ou compagnon de solitude. S'agit-il d'un homme ou d'une femme? Laissons la question ouverte pour le moment.

Entre la première et la dernière strophe, qui est la même, le narrateur raconte, dans le détail, son itinéraire de vagabond ou de «pélerin de la conquête». Un matin, semble-t-il dire, j'ai décidé de quitter ma chambre et de chercher partout l'Introuvable: a) sur les routes... que d'autres pas avaient déjà réduites en poussière; b) dans une auberge: vin rouge, crimes, face pâle (assiettes) de vagabonds illuminés tombés là au bout de leur rêve; c) «au carrefour d'un vieux village sans amour"; d) "dans les grincements des express où les silences des arrêts s'emplissaient des noms des stations"; e) "dans une plaine où des étangs s'ouvraient au ciel tels des yeux clairs»; f) «dans les livres qui sont des blancs laissés en marge de la vie", des notes confuses prises à l'écoute “de la conférence des choses"; g) mais un jour, son cœur qu'il avait cru mort fut «surpris par un retour de flamme"; un jour en effet, après le départ tout récent d'un navire, il a failli «saisir la forme enfuie d'une autre main», mais sa main ne se referma que sur "l'affreux vide d'elle-même", autrui restant l'absent; h) dans les yeux d'autrui, ceux qui le dévisageaient, ceux qui lui manifestaient de la haine. Mais pourquoi le hairait-on? Quelle raison le narrateur devine-t-il? Bref, continue le narrateur sans s'expliquer, j'ai dû me résigner à revenir et à venir vous supplier de m'ouvrir et de me faire l'aumône de la clarté, car, 
Je ne savais plus, du pays,

Mériter une paix échue

Des choses simples et bien sues.

Trop de fumées ont enseigné

Au port le chemin de l'azur,

Et l'eau trépignait d'impatience

Contre les portes des écluses.

Les poèmes $1 \mathrm{I}^{41}$ et $1 \mathrm{II}^{42}$ contiennent les deux seules images verticales de Loranger: a) celle du cerf-volant et du fil raidi qui le retient à la terre; et b) celle du sablier et de la chute du sable de haut en bas... et que l'on retourne périodiquement. Mais il ne faut pas s'y tromper. Encore une fois, l'imaginaire de Loranger semble organisé unidimensionnellement. L'imaginaire occidental est un espace et la durée s'exprime en distance: temps court, long, etc. Chez Loranger, on l'a vu, tout se passe comme si l'horizontal était la dimension fondamentale. Le cerf-volant est prétexte à un motif obsédant, celui de «la route qu'on étire». Le sablier est prétexte aussi à une méditation sur le temps: rêver l'avenir, c'est le chemin par excellence pour saisir le passé.

Dans le poème II, le désir se trouve «rassasié» puisque le narrateur est en mouvement, en marche. Mais ce qui vient tout à coup brouiller le plaisir qu'il éprouve de sa course, c'est la route qu'il «déroule" depuis son départ, celle qu'il "étire " derrière lui. Ressemblant à un fil attaché quelque part dans le passé, la route appelle paradoxalement l'image verticale du cerf-volant: au bout du chemin, notre marche se raidit soudain comme un fil de cerf-volant qui rappelle à la terre "l'incontrôlable ascension, l'immense besoin d'azur". Au plus haut de sa course, le cerf-volant tend le fil et rappelle à celui qui le retient, au point fixe, l'incontrôlable soif d'aller toujours plus haut, plus loin. Mais la problématique du poème relève de la temporalité, non de l'espace: il manque au narrateur cette sensation d'un fil qui se raidirait, attaché qu'il serait à son passé. Au contraire, la route se «déroule» et "s'étire» sans point d'attache et sans frontière, comme une dérive.

Le poème III choisira un symbole spatial qui aura moins d'amplitude, un symbole qui correspondra davantage à l'image que l'on se fait communément du déroulement du temps, de l'écoulement du temps. II s'agit du sablier (moins utilisé par Loranger que le cadran rond de l'horloge) et des nombreuses connotations qu'il a pu rassembler en un très court texte: égrènement du temps, chute sans fin des "espoirs pulvérisés", entassement, alourdissement et, sur le plan psychologique accablement, l'inverse de la trop grande légèreté du cerf-volant...

L'avenir n'est rien qu'un retour

Perpétuel sur soi-même,

La vie qu'on reprend à l'inverse,

Un passé toujours ressassé

Comme un sablier qu'on retourne.

D'où la moralité de l'Invitation au retour ${ }^{43}$ : «Reviens au pays [...] où dort ton passé sous la cendre. " Par rapport au passé, les poèmes II et III sont 
contradictoires: a) besoin d'une attache pour que la route ait un but ou un sens; et b) hantise du passé comme un poids lourd, comme un retour pénible sur sa propre lourdeur. L'avenir, c'est en effet le sablier qu'on retourne, le jeu des mêmes grains de sable...

Le thème majeur des poèmes de Loranger, c'est sûrement la hantise du voyage. Cependant, l'expérience amène le narrateur à métamorphoser cette hantise du mouvement en une méditation posée sur le temps: rêverie immobile sur la présence du temps (silence, ombre, solitude, rondeur de l'intimité...). D'où la moralité qu'il retire de sa sagesse toute naïve: rêver l'avenir, c'est revivre le passé. Comme pour le passeur, l'espace en général ou le mouvement en particulier prend d'autant plus d'importance que la sensation de la durée s'impose à lui, sensation puisque la vieillesse s'éprouve à travers la redécouverte des bras, d'une tête, des reins (qu'on attrappe comme une maladie) jusqu'à la paralysie. La vieillesse est alors une nouvelle vie, le passé revécu en un présent immobile et fait d'«espoirs pulvérisés".

Le poème IV 44 n'est autre qu'une variation de la parabole biblique de l'enfant prodigue, des départs conquérants et des retours déçus et repentants. Dans le poème $\mathrm{VI}^{45}$, le rêveur retrouve sa stabilité, son immobilité et son attitude de témoin:

Je suis stable, maintenant,

Circonscrit dans un exergue

Qu'est ce grand mur tout autour

De la maison du retour

Que m'importe l'horizon

[...] Qu'il recule toujours.

Mais chez cet observateur quasi objectif de l'espace, cohabite le rêveur qui médite sur le temps: la route fut longue et l'absence prolongée, mais

Comme tout cela est court,

Quand je le vois par la fin.

II ne se souvient pas d'avoir été vagabond. II ne lui reste plus qu'une "mémoire lasse" pour recenser vainement "un passé rapetissé", "des passés qui s'interceptent», ceux même du voyage, et ceux, retrouvés, d'avant le voyage. Bref, la distance parcourue et le temps perdu à vouloir laisser mourir le passé... rien de mieux, à l'arrêt, pour voir bondir de nouveau ce même passé, en un mouvement fou d'aller-retour ou de va-etvient.

\section{L'ABSENTE}

Jamais il n'a été question de la femme jusqu'ici. Exception faite des deux derniers vers de Serti dans ton souvenir, dernier poème du recueil du Retour de l'enfant prodigue, la femme est absente chez J.-A. Loranger, absente dans les Atmosphères et à peine évoquée dans Poèmes. Le poème 
V46 a été volontairement négligé par nous. Il est en fait une reprise du texte préliminaire des Poèmes, avec cependant d'importantes variantes temporelles au début et à la fin des deux textes. "Que deviendra mon cœur / Desserti de ton amour... ${ }^{47}$, écrira-t-il au début du recueil, avant toutes les rêveries de partance de Marines. "Que serais-je devenu / Desserti de ton amour", reprendra l'enfant prodigue à son "retour repenti", privé des “joies accessibles". C'est pratiquement l'unique poème (répété) dans lequel Loranger semble évoquer une présence féminine. Si tel était le cas, la femme apparaîtrait implicitement comme celle qui habite la chambre; elle pourrait être associée à toute une thématique de la lampe, des insomnies et des rêveries sans cesse reprises. On reconnaîtrait là, en filigrane, l'angélisme des Nelligan, Loseau ou A. de Bussières. Cependant, la femme demeurerait surtout comme quelqu'un vers qui l'on revient, et l'enfant prodigue, contrairement au personnage biblique, ne reviendrait pas tout repenti vers son père, mais vers une femme, sinon sa mère: "Tel obstiné à l'espoir / Le Père attend le Prodigue, / Ainsi, pieusement, mon cœur / Serti dans ton souvenir, "Comme chez Nelligan, le père serait associé au voyage tandis que la mère ou la femme en général se trouverait étroitement liée à la chambre, la lampe et la page blanche. Elle serait donc rêvée comme une compagne du rêve et de l'écriture. Comme G. Bessette a réussi une psychanalyse des textes de Nelligan, il serait tentant d'appliquer les mêmes modèles aux poèmes de Loranger. Comme chez Nelligan, la femme chez Loranger, même si elle est très peu évoquée, apparaîtrait associée à des objets ronds et creux (anneaux, cercle lumineux de la lampe, kiosque, etc.) ou à des instruments de musique comme le disque d'un phonographe (mélodies vaines et figées dans la cire des disques) ou encore la voix d'un vieux piano qui "garde enclos / Comme une momie, / L'accent de [son] ccur brisé".

Mais la femme n'est jamais présente en chair et en os. Jamais elle n'est regardée et encore moins touchée. C'est une absente... regrettée, mais impuissante à le distraire de “l'appel ému des sirènes". Elle reste un souvenir, celle vers qui l'on revient, celle qui demeure attachée pour toujours à une breloque en or usée par le temps. Pour tout dire, l'enfant prodigue ne peut parler que d'un père, d'un fils ou d'un amant. L'autre, si rarement évoqué, est un compagnon que l'on regrette de quitter ou vers qui l'on revient après une fugue décevante. C'est du moins à cette conclusion que nous convie le texte:

La breloque, dont s'éteint

Au souvenir, le vestige

Qu'elle avivait du passé,

Ne vaut que son pesant d'or,

Au plateau de la balance.

Et pour t'avoir tant aimé,

Enchassé dans ton étreinte,

Ce cour, que tu désavoues(ais),

Ne se rajeunira pas (N'allait pas se rajeunir)

De l'or dont il est (était) usé ${ }^{48}$. 
Désir obsédant et impuissant de départ, situation d'attente patiente et morose, valorisation de l'exil intérieur, cela suffit pour reconnaître les textes de Loranger comme un témoignage vrai et symptomatique d'un certain type de production littéraire au début du siècle au Québec. Toutefois, Dieu est absent chez Loranger. Même chose pour la femme. Le désir d'errance du poète ne mène nulle part. $A$ ce vagabondage décevant, l'écriture apportera un dérivatif salvateur: au cœur même de sa solitude, l'écrivain circonscrit les froṇtières de sa rêverie. L'errance se poursuit alors entre les quatres murs d'une chambre parquée de silence et de solitude. En ce sens, Loranger n'est pas de son époque. Poète "artiste" comme on aimait alors nommer ceux qui ne vantaient pas nos valeurs nationalistes, sinon régionalistes, les textes de Loranger demandent à être lus non pas à la lumière de l'École littéraire de Montréal, mais à celle projetée par la production moderne des Saint-Denys Garneau, Anne Hébert, Alain Grandbois... Les lecteurs des années 20 n'ont pas su comprendre Loranger. II aura fallu attendre cinquante ans pour qu'on sache en apprécier l'originalité et, en même temps, l'appartenance à une conscience collective toujours en retard sur la clairvoyance profonde des vrais poètes. Au Québec, notre critique a trop longtemps confondu poésie et versification.

Robert Giroux

Université de Sherbrooke

1. Études françaises, vol. $3, n^{\circ} 3$, août 1967, p. 303-304. Numéro spécial consacré à Nelligan.

2. Ibid., p. 304.

3. Gilles Marcotte, Avant-propos, les Atmosphères suivi de Poèmes, Montréal, HMH, "Sur parole", 1970, p. 16. Toutes nos citations des textes de Loranger sont extraites des Atmosphères. La provenance des autres citations sera toujours indiquée: le Village, contes et nouvelles du terroir, Montréal, E. Garand, 1925; Récits, dans les Ecrits du Canada français, $n^{\circ} 35$, Montréal, $\mathrm{HMH}, 1972$. De Terra Nova, J. Fournier donne un extrait dans son Anthologie des poètes canadiens (1920) et B. Brunet dans son Histoire de la littérature canadienne (1946, réédition, $\mathrm{HMH}, 1970$ ). En appendice à sa thèse de doctorat, $B$. Guilmette propose aussi de nombreux textes de Loranger, en plus de fournir une bibliographie exhaustive de l'écrivain. Nous n'analyserons que les recueils publiés par HMH, les autres textes devant être étudiés plus tard.

4. Les Atmosphères, Moments, V, p. 103-104.

5. Ibid., p. 27-45.

6. "Un clocher n'est jamais seul dans la plaine", la “Long Trail», dans Écrits du Canada français, $\mathrm{n}^{\circ} 35,1972$.

7. Les Atmosphères, p. 65-71.

8. Dans l'Eau et les rêves (Paris, J. Corti, 1942, p. 97 à 113), Bachelard a de belles pages sur le complexe de Caron. L'ustensilité du traversier n'est pas suffisante pour déterminer ou légitimer le risque ultime du passeur à tenter une dernière traversée. Des intérêts puissants le poussent, profonds et fabuleux, pressé par des aspirations ou des besoins chimériques qui seuls légitiment l'aventure, la traversée, la mort, moins acceptée que désirée. Peut-il désirer plus belle mort? Après le va-et-vient coutumier, la dérive, la noyade, l'abandon à la directive des eaux... "ll y a toujours de l'eau quelque part", serat-il dit par Loranger dans le récit du Dernier des Ouellette, Ecrits du Canada français, $n^{\circ} 35,1972$. 
9. Loranger évoquera souvent çette figure du vagabond: Kenoche, le quêteux de La Noroua, qui se pendra (Écrits du Canada français, $n^{\circ} 35,1972$ ) et surtout la figure biblique de l'enfant prodigue qui, d'abord jeune "pélerin de la conquête " devient ce "vagabond cherchant ses traces dans le vent ".

10. Les Atmosphères, Signets, p. 55.

11. Ibid., p. 54.

12. Ibid., p. 56.

13. Ecrits du Canada français, $n^{\circ} 35,1972$.

14. J. Fournier, Anthologie des poètes canadiens, 1920.

15. B. Brunet, Histoire de la littérature canadienne, Montréal, HMH, 1970, p. 216.

16. Les Atmosphères, Marines, p. 77.

17. Ibid., p. 78.

18. Les Atmosphères, le Passeur, p. 45.

19. Les Atmosphères, Marines, p. 79.

20. Ibid., p. 80-82.

21. Ibid., p. 83-85.

22. Ibid., p. 89-92.

23. Les Atmosphères, Moments, XI, p. 115.

24. Les Atmosphères, Marines, p. 86-88.

25. Ibid., p. 82. Serait-ce les profondeurs marines?

26. Les Atmosphères, Moments, IX, p. 111.

27. Ibid., p. 95.

28. Journal de Saint-Denys Garneau, Montréal, Fides, 1949, p. 136.

29. Les Atmosphères, Marines, p. 89-92.

30. Les Atmosphères, Moments, p. 95-96.

31. Les Atmosphères, Moments, IX, p. 112.

32. Les Atmosphères, Moments, III, p. 99.

33. Ibid., p. 100. Sur la thématique de la chambre (intimité), des portes et du corridor (extérieur), voir les pages $55,99,131$.

34. Les Atmosphères, Marines, p. 91.

35. Les Atmosphères, Moments, IV, p. 101-102.

36. Ibid., p. 103. Voir aussi, p. 117, le douzième moment où “La lampe casquée / Pose un rond sur l'écritoire / - Une assiette blanche.» Et à la p. 132, les assiettes rappellent «la face pâle / Des vagabonds illuminés / Tombés là au bout de leur rêve. " Et au sixième moment (p. 106) : «Pour toute la peine / Dont se gonflait mon poëme». Et si l'on passe de l'intérieur à l'extérieur, au dehors, on a le dix-huitieme moment (p. 125-126): «Le soir pense dans son ombre / Comme des yeux clos, / Des pensées tristes lui naissent / Comme des hiboux. / - J'avoue la nuit et l'attente. / Premier quartier de la lune / Son disque d'argent / Que vient de planter au ciel, / Soudain réveillé, / Le beau discobole antique." Et à la page 134: “Dans une plaine où des étangs / S'ouvraient au ciel tels des yeux clairs. "Ou encore le septième moment ( $p$. 107): "Au pied des grands arbres, / L'ombre est endormie en rond." Enfin, le huitième moment (p. 110): "Et sous les chocs de mes pas, / Dans l'allée du parc, / Je me désarticulai, / Pareille à la caisse / Qu'on fait rouler sur ses angles."

37. Les Atmosphères, p. 151.

38. Histoire de la littérature canadienne, p. 218.

39. Les Atmosphères, p. 134.

40. Les Atmosphères, Signets, p. 55.

41. Les Atmosphères, p. 137-138.

42. Ibid., p. 139-140.

43. Ibid., p. 149-150.

44. Ibid., p. 141-143.

45. Ibid., p. $146-148$.

46. Ibid., p. 144-145.

47. Ibid., p. 75.

48. Ibid., p. 145. La femme est cependant un acteur dominant dans les contes ou les nouvelles de Loranger, personnage dominant par la force de son caractère, la ruse ou l'obstination. Relire l'Orage, la Revanche, De miraculeuses matines, la Savane des Cormier, le Garde forestier (où c'est l'Indienne qui est le personnage déterminant du récit), et Mrs. Carry-Nations (Écrits du Canada français, $n^{\circ} 35,1972$ ). Nous espérons pouvoir revenir là-dessus à l'occasion d'un autre article. 